\title{
CLIMA FAMILIAR Y RENDIMIENTO ACADÉMICO EN ESTUDIANTES DEL CENTRO DE IDIOMAS DE LA UNIVERSIDAD NACIONAL JOSÉ MARÍA ARGUEDAS - ANDAHUAYLAS 2018
}

\author{
FAMILY ENVIRONMENT AND ACADEMIC PERFORMANCE IN \\ STUDENTS FROM THE LANGUAGE CENTER OF THE NATIONAL \\ UNIVERSITY JOSÉ MARÍA ARGUEDAS - ANDAHUAYLAS 2018 \\ (D) Yolanda Aroquipa Durán ${ }^{1 *}$, (iD Reynaldo Sucari León ${ }^{1}$ \\ yolandaaroquipa@gmail.com; reysucari@gmail.com \\ ${ }^{1}$ Universidad Nacional Autónoma de Huanta, Ayacucho, Perú
}

*Correspondencia: Yolanda Aroquipa Durán. Email: yolandaaroquipa@gmail.com

Recibido: 05.07.19 | Aprobado: 01.12.19

\section{RESUMEN}

La investigación tuvo como propósito fundamental determinar la relación que existe entre clima familiar y rendimiento académico en los estudiantes de dicho centro de estudios, que fue guiada por la hipótesis general declarando que existe relación directa y significativa entre clima familiar y rendimiento académico en estudiantes del Centro de Idiomas de la Universidad Nacional José María Arguedas de Andahuaylas en el año 2018. Es una investigación de tipo básica, nivel descriptivo y diseño no experimental de corte transversal, en la que se ha empleado el método científico y estadístico para alcanzar los resultados esperados, el estudio consideró una muestra estratificada de 90 estudiantes; cantidad determinada mediante muestreo probabilístico de una población de 117 estudiantes matriculados en el Centro de Idiomas de la Universidad Nacional José María Arguedas en los niveles básico e intermedio durante el primer semestre académico del 2018. Concluyendo que con un nivel de significancia del 5\% se afirma que existe una relación directa y significativa fuerte entre clima familiar y rendimiento académico en estudiantes del Centro de Idiomas de la Universidad Nacional José María Arguedas de Andahuaylas, dado que el coeficiente de correlación alcanza un valor de 0.778; estableciendo que, cuanto mejor es el clima familiar mejor será el rendimiento académico de los estudiantes respecto al idioma inglés.

Palabras clave: Centro de idiomas, clima familiar, rendimiento académico.

\begin{abstract}
The research had as main purpose to determine the relationship between family environment and academic performance in the students of this study center, which was guided by the general hypothesis declaring that there is a direct and significant relationship between family environment and academic performance in students from the Language Center of the National University José María Arguedas de Andahuaylas in 2018. It is a basic research, descriptive level and non-experimental design of cross section, in which the scientific and statistical method has been used to achieve the expected results, the study considered a stratified sample of 90 students; amount determined by probabilistic sampling of a population of 117 students enrolled in the Language Center of the National University José María Arguedas at the basic and intermediate levels during the first academic semester of 2018. Concluding that with a level of significance of $5 \%$ it is affirmed that there is a direct and significant strong relationship between family environment and academic performance in students of the Language Center of the National University José María Arguedas of Andahuaylas, insomuch as that the correlation coefficient obtains a value of 0.778 ; establishing that, as much as better is the family environment, better will be the students' academic performance with respect to the English language.
\end{abstract}

Keywords: Language center, family environment, academic performance. 


\section{INTRODUCCIÓN}

Estudios realizados por Moreno (2009) sostienen que el clima familiar se relaciona de manera directa con el clima social del aula, en la que se ha observado la calidad del clima familiar mediante el desarrollo de la empatía, la actitud del profesorado, y el comportamiento violento de los adolescentes. También por su parte Begoña (2010) centró su investigación en la percepción del clima familiar en toxicómanos concluyendo que el clima familiar tiene una influencia significativa en todas las esferas de su desarrollo personal y social de los integrantes de las familias y más aún en la juventud. Estudios similares, realizados por Rosales (2013) respecto a la percepción del clima familiar en adolescentes de diferentes tipos de familias (nucleares, uniparentales, extensas y reconstituidas) todos ellos estudiantes de la Universidad Autónoma de México, afirma que independientemente del tipo de familia que constituye, el clima social familiar puede ser adecuado para el desarrollo de sus integrantes, pues a pesar de las transformaciones sociales, la familia puede proveer condiciones adecuadas para todos sus miembros que les garantizará la supervivencia. Otro estudio realizado por Estevez (2008) sostiene que existe una asociación directa entre el clima escolar frente a la satisfacción vital del adolescente, así mismo el clima social familiar ejerce una influencia directa sobre el ánimo depresivo de los estudiantes.

También Gonzales y Pereda (2009) por su parte realizaron una investigación sobre el clima social familiar y el rendimiento escolar en estudiantes de educación primaria, llegando a concluir que, efectivamente, el clima social familiar se relaciona de manera significativa con el rendimiento escolar, dado que existe un alto grado de correlación. De manera específica, el $63.33 \%$ de estudiantes presentan un nivel inadecuado del clima social familiar; así mismo, un $74.67 \%$ de estudiantes tienen un aprendizaje regularmente logrado con un promedio de 12 puntos como promedio. Estos resultados indican que el clima social familiar si es una condicionante para el rendimiento escolar en los estudiantes de educación primaria. Así mismo Chávez (2011) en una investigación cuyo propósito fue establecer la relación del clima familiar y el rendimiento académico en los estudiantes, llegan a la conclusión de que efectivamente existe relación directa y significativa entre el clima familiar y el rendimiento académico, ya que el coeficiente de correlación " $\mathrm{r}$ " de Pearson es de 0.89 a un nivel de significancia del 5\% en el estudio. Todo ello indica claramente que un buen clima familiar hará que se consiga un alto rendimiento académico en los estudiantes.

Respecto a las bases teóricas del clima familiar, se tienen los aportes de García (2005) cuando indica que "el clima social familiar está relacionado con las interacciones que los padres desarrollan con los hijos en el hogar, estas pueden ser cantidad y calidad". Ello indica que cuando se hace referencia al clima social familiar también se hace referencia a los factores materiales, espirituales, psicológicos, sociales y culturales que intervienen en la calidad de las interacciones entre todos los miembros de una familia. En síntesis, el clima social familiar es la apreciación de las características socio ambientales de una familia, todos estos son descritos en función de las relaciones interpersonales de los miembros de dicha familia, todo aspecto de desarrollo cobra mayor importancia en ella y su estructura que lo conforma. 
Así mismo, el constructo familia, según la Enciclopedia interactiva Encarta (2009), es el grupo social básico y fundamental creado por vínculos de parentesco o matrimonio en todas las sociedades, luego también añade a ello, toda familia proporciona a sus miembros integrantes protección, compañía, seguridad y socialización. También por otra parte, Sloninsky (1962) hace referencia sobre la familia, indicando que es un organismo que cuenta con una unidad funcional, así como, está en relación al parentesco, la veracidad y la sociedad, creando entre sus miembros influencias e interacciones mutuas. La estructura interna de toda familia determina la formación y el grado de madurez de todos sus integrantes. Apoyando ello Benites (1997) sostiene que la familia aun es como la estructura básica de la sociedad, con funciones que hasta aún no han sido sustituidas por otras organizaciones creadas expresamente para asumir dichas funciones. De estas, la más importante es aquella que hace referencia a servir como agente socializador que permite proveer condiciones y experiencias vitales que facilitan un desarrollo óptimo en el aspecto biopsicosocial de sus hijos. El clima social familiar es evaluado empleando una escala adecuada para dicha variable, que se halla sustentada por Moos y otros (1989) cunado detallan la escala para medir el clima social familiar.

Respecto al rendimiento académico, se consideró aportes teóricos realizados por Suárez (1998) cuando afirma que el rendimiento académico muestra el resultado de muchas y complejas faces en el proceso educativo y, al mismo tiempo, una de muchas metas que convergen en los esfuerzos de los estudiantes, las iniciativas de las autoridades, los maestros, los padres de familia y los estudiantes. En este sentido, el rendimiento académico es considerado como un conjunto de transformaciones realizadas en el estudiante, ocasionado por diversos factores en el proceso de enseñanza y aprendizaje, manifestándose mediante el crecimiento y enriquecimiento de la personalidad en formación. Por su parte, Palomino (2002) indica que el rendimiento académico sintetiza la acción del proceso educativo, que no solo representa el aspecto cognitivo logrado por el estudiante, sino también están inmersos todo un conjunto de habilidades, destrezas, aptitudes, ideales, intereses y otros similares. Todo ello se puede conseguir gracias al esfuerzo de la sociedad, del profesor, de las autoridades, de los padres de familia, así mismo hermanos y hermanas dentro del núcleo familiar.

También el rendimiento académico desde la óptica del docente es entendido por Solier (2008) como una medida de los indicativos que se manifiestan en forma estimativa de lo que una persona ha aprendido como consecuencia de un proceso de formación o instrucción. El mismo autor tomando en cuenta la perspectiva del estudiante define el rendimiento académico como la capacidad adquirida que evidencia los objetivos educativos pre establecidos, los cuales están explícitamente detallados en el DCN 2009, propuesto por el Ministerio de Educación.

Novaez (1986) indica que el rendimiento académico es un quantum alcanzado por el individuo en una determinada actividad académica, ella está ligado más a la aptitud y además de ello es el resultado de factores volitivos, afectivos y emocionales logrados a través de la ejercitación. En todo ello cabe mencionar que Peñaloza (2004) denomina al rendimiento académico hoy en día como un aprendizaje cognitivo. Así mismo el quantum se refiere a una cantidad mesurable que todo estudiante puede obtener en un sistema específico, en el caso peruano es el sistema vigesimal de 0 a 20 puntos. 
Por otra parte, el idioma inglés se ha convertido, en la actualidad, en una lengua internacional cuyo aprendizaje es requerido para que los estudiantes tengan mejores oportunidades en su proceso de formación ya sea básica o superior, ello se realiza con posibilidades de aprovecharla para conseguir satisfacer las necesidades de comunicación, estudio, trabajo, viajes y entre otros muy particulares. Así mismo, el aprendizaje del idioma inglés permite a los estudiantes acercarse a las últimas novedades en cuanto a la ciencia y la tecnología, todos ellos publicados en su mayor parte en idioma inglés, que es necesario seguir aprendiendo a lo largo de su vida y continuar con estudios en el nivel superior.

Lumman (2006) indica que el rendimiento académico en el idioma inglés se sustenta bajo tres competencias: (1) expresión y comprensión oral, (2) comprensión de textos y (3) producción de textos.

a. Expresión y comprensión oral: Ello implica el desarrollo interactivo de las capacidades dentro de la comprensión y producción de textos a nivel oral. Este proceso se efectiviza en diversas situaciones comunicativas y bajo diversos propósitos relacionados con la vida cotidiana ya sea en el entorno familiar o social del estudiante. Esta competencia involucra el saber escuchar, así como expresar las propias ideas emociones y sentimientos en diversos contextos y con diferentes interlocutores.

b. Comprensión de textos: La capacidad de comprensión de textos considera la reconstrucción del sentido del texto, que es un proceso que permite distinguir las ideas principales, así como las secundarias, teniendo en cuenta las estructuras lingüísticas apropiadas al texto. Ello facilita la recepción crítica de la información para una adecuada interacción comunicativa y para obtener nuevos aprendizajes.

c. Producción de textos: En la producción de textos se desarrolla el proceso que conlleva poder expresar las ideas, emociones y sentimientos en el marco de una reestructuración de los textos que fueron planificados. Ello motiva el espíritu activo y creador y, además, facilita el manejo adecuado de los códigos lingüísticos y no lingüísticos. Estos conocimientos sirven como soporte para desarrollar la competencia comunicativa, además están organizados en léxico, fonética, recursos no verbales y gramática.

Por todo ello, en la presente investigación se pretende dar solución al problema ¿qué relación existe entre clima familiar y rendimiento académico en estudiantes del Centro de Idiomas de la Universidad Nacional José María Arguedas de Andahuaylas en el año 2018?

\section{METODOLOGÍA}

La presente investigación considera dos variables como objetos de estudio; el clima familiar y el rendimiento académico en el idioma inglés, así mismo en la investigación se ha empleado el método científico como método general, dado que dicho método comprende un conjunto de normas que regulan el proceso de cualquier investigación que merezca ser calificada como científica, así también se ha empleado el método estadístico, ya que ello permitió describir los datos y características de la muestra de estudio. 
La investigación es de tipo básica; dado que es un estudio que describe hechos y fenómenos tal como son, es de nivel descriptivo - correlacional, y sigue un diseño no experimental; así mismo, es un estudio de corte transversal, dado que el recojo de datos se da en un solo momento del tiempo. En cuanto a la población de estudio estuvo conformada por 117 estudiantes matriculados en el Centro de Idiomas de la Universidad Nacional José María Arguedas de Andahuaylas, ello en el primer semestre académico 2018, de todo ello mediante el muestreo probabilístico se obtuvo una muestra representativa de 90 estudiantes tanto del nivel básico e intermedio.

Para el recojo de datos se ha empleado un cuestionario de encuesta sobre el clima familiar y una prueba escriba para medir el rendimiento académico en idioma inglés; ello generado por el software ID English en su respectivo nivel (básico o intermedio) considerando las tres competencias fundamentales para el idioma de inglés. Estos dos instrumentos fueron validados mediante juicio de expertos, así mismo mediante una muestra piloto de 15 estudiantes se ha encontrado el coeficiente de confiabilidad obteniéndose un valor de alfa de Crombach de 0.780 y 0.802 para los instrumentos, deduciendo que dichos instrumentos de investigación tienen una excelente confiabilidad.

En cuanto a las técnicas de procesamiento y análisis de los datos se ha empleado las tablas de distribución de frecuencia (absolutas y porcentuales) por las cuales han sido procesados los ítems de los dos instrumentos, obteniéndose los gráficos de barra que mejor representan los resultados, asimismo se ha calculado el coeficiente de correlación " $r$ " de Pearson con su respectiva prueba de hipótesis para la significancia correspondiente, mediante la distribución " $\mathrm{t}$ ” de Student así como el "p-value” a nivel de probabilidades.

\section{RESULTADOS}

La investigación presenta como resultados la medición a dos variables de estudio; clima familiar y rendimiento académico, ello a través de los instrumentos aplicados a 90 estudiantes del Centro de Idiomas de la Universidad Nacional José María Arguedas de Andahuaylas durante el primer semestre del año 2018.

El aspecto descriptivo de la variable clima familiar, presenta la siguiente información:

\section{Tabla 1.}

Resumen general por dimensiones del clima familiar en los estudiantes

\begin{tabular}{llllll} 
& Estadígrafos & Relaciones & Desarrollo & Estabilidad & Promedio \\
\hline & Suma & 1355 & 2573 & 1161 & 1696.33 \\
& Media & 14.99 & 28.46 & 12.85 & 18.7719 .00 \\
& Mediana & 15 & 29 & 13 & 19.6725 .33 \\
& 15 & 29 & 15 & 10.00 \\
& 21 & 38 & 17 & 15.33 \\
& Moda & 17 & 6 & 2.96 \\
& Puntaje máximo & 21 & 11 & 9.44 \\
& Asimetría & -0.02 & -0.39 & -0.2 & -0.20 \\
& Kurtosis & -0.12 & 0.01 & 0.09 & \\
\hline
\end{tabular}




\section{Figura 1}

Comparación de estadísticos descriptivos por dimensiones del clima familiar

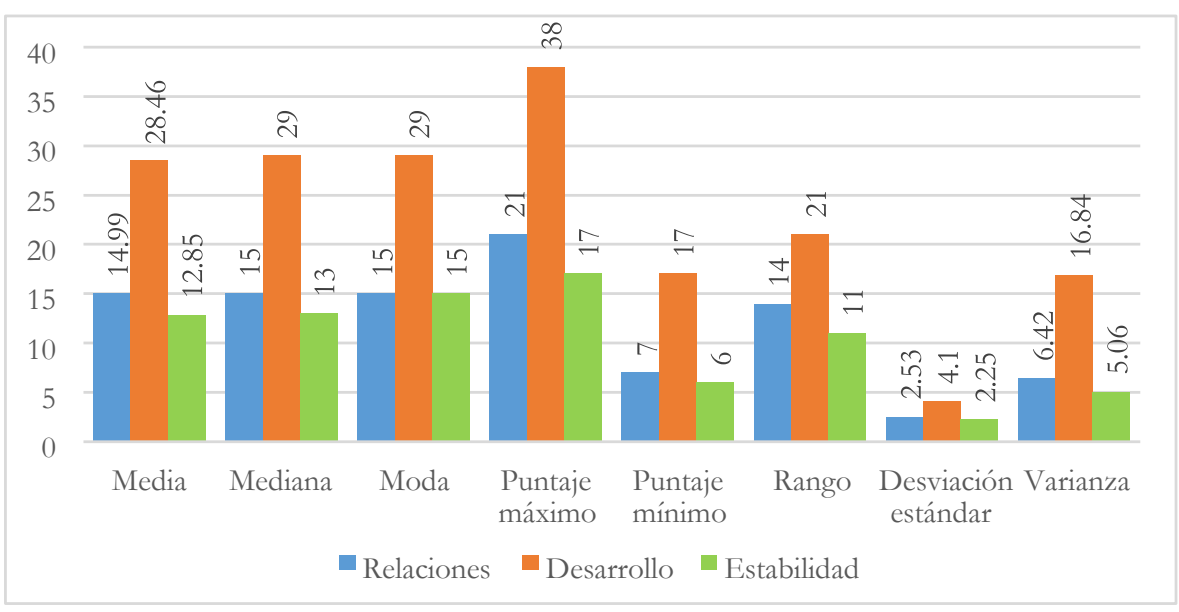

La tabla 1 y figura 1 muestran a la variable clima familiar, donde se aprecia que cuenta con tres dimensiones, en ello se observa que la dimensión desarrollo alcanza las puntuaciones más altas, en cambio las otras dos; relaciones y estabilidad se mantienen casi en la misma proporción, y entre las tres dimensiones la dimensión estabilidad presenta valores más homogéneos, indicando que los estudiantes mantienen una convergencia respecto a esta dimensión; es decir que tienen puntajes muy similares, seguido de la dimensión relaciones, en cambio la dimensión desarrollo presenta una variación mayor.

El aspecto descriptivo de la variable clima familiar, presenta la siguiente información:

\section{Figura 2}

Nivel del clima familiar en los estudiantes

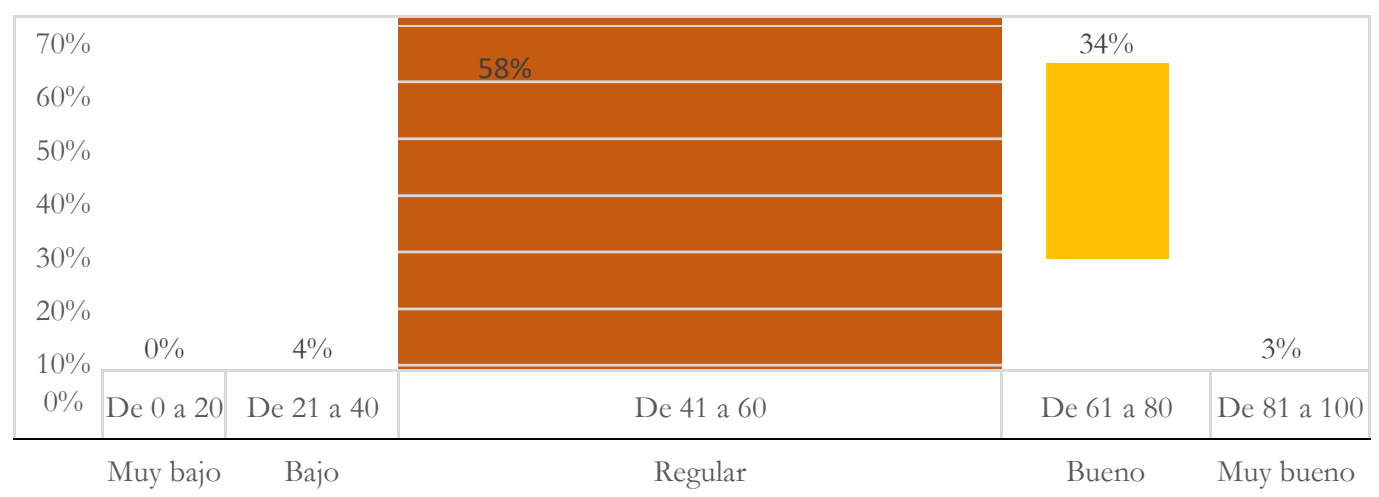

La figura 2 muestra los resultados a nivel global del clima familiar en el cual el 58\% de los estudiantes encuestados indican contar con un clima regular dentro de la familia, y un 34\% indican que se desarrollan dentro de un clima familiar bueno, inclusive se cuenta con un 3\% de estudiantes que se desenvuelven dentro de un clima familiar muy bueno, y por la parte contraria también existe un $4 \%$ de estudiantes que cuentan con un clima bajo dentro de sus familias. En todo ello se deduce que el nivel predominante del clima se encuentra en la opción regular; considerado como favorable y que aún puede ser 
mejorado, permitiéndole a los estudiantes un óptimo desarrollo en su vida académica y social.

Por otra parte, los resultados respecto a la variable rendimiento académico fueron medidos por una prueba escriba para medir el rendimiento académico en idioma inglés; dicha prueba fue generada por el software ID English en su respectivo nivel (básico o intermedio) considerando las tres competencias para el idioma de inglés; como son: expresión y comprensión oral comprensión de texto y producción de textos. Así el aspecto descriptivo de la variable rendimiento académico presenta lo siguiente:

\section{Figura 3}

Nivel del rendimiento académico en idioma inglés

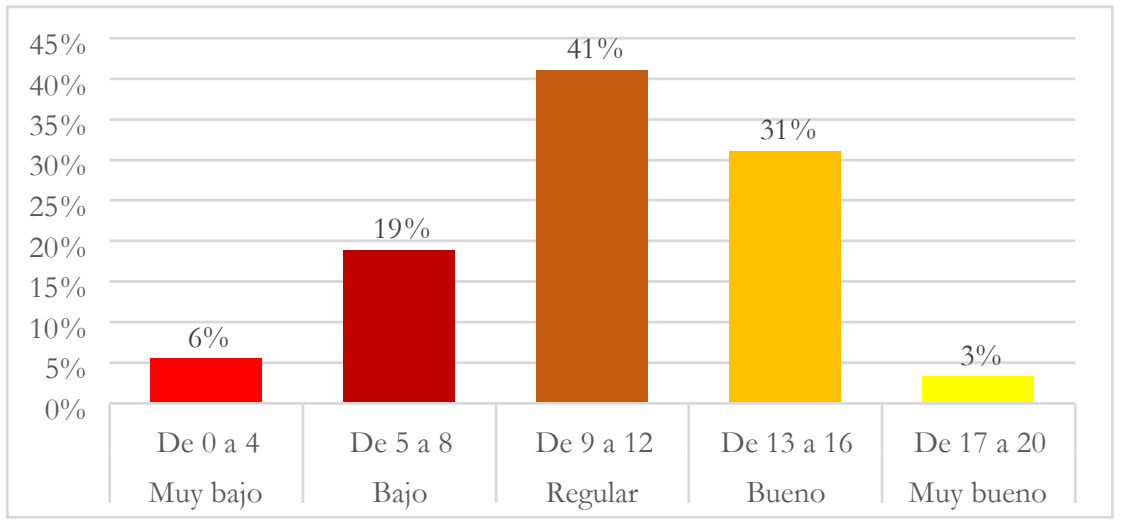

En la figura 3 se observa la distribución que presenta los resultados de la variable rendimiento académico, observando en ello que el $41 \%$ de los estudiantes tienen un rendimiento regular, acompañado de un 31\% que presentan un buen rendimiento, así mismo un escaso $3 \%$ de estudiantes logran un rendimiento académico muy bueno en el idioma inglés, pero por parte contraria se tiene a un $19 \%$ de estudiantes que aún muestran dificultades en su aprendizaje presentando un rendimiento bajo y más aún un $6 \%$ necesita de manera urgente una atención; ya que se encuentran en el nivel muy bajo.

También se puede apreciar los estadísticos descriptivos de la variable rendimiento académico en el idioma inglés, siendo ellos:

\section{Figura 4}

Estadísticos descriptivos del rendimiento académico en idioma inglés

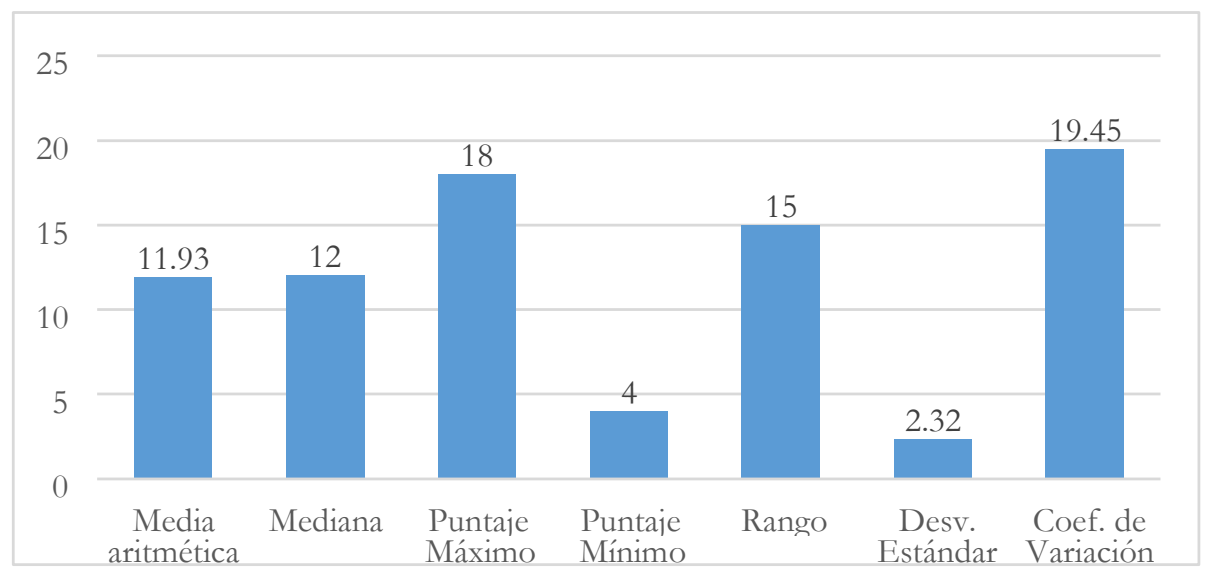


Según la figura 4 claramente se puede apreciar que la variable rendimiento académico en el idioma ingles que fue medido a 90 estudiantes del Centro de Idiomas de la Universidad Nacional José María Arguedas de Andahuaylas durante el primer semestre 2018, muestra un valor promedio de 12 puntos, así mimo la mediana alcanza un valor de 12 puntos ello indica que el $50 \%$ de estudiantes tienen una nota menor o igual a 12 puntos y el resto tiene una nota mayor a los 15 puntos, de todos los estudiantes hay estudiantes que alcanzan un resultado sobresaliente hasta de 18 puntos, pero también aún existen estudiantes con un puntaje muy bajo de 4 puntos, a pesar de todo ello en la distribución hay una desviación estándar de 2.32 puntos y un coeficiente de $19.45 \%$; estos dos últimos indican que la distribución de datos corresponde a una muestra homogénea.

Como tercer punto presentamos el contraste de la hipótesis, para ello se ha empleado el software SPSS v.24 en el cual permite automatizar procedimientos de cálculo estadístico, mostrando el siguiente resultado:

\section{Tabla 2}

Coeficiente de correlación entre clima familiar y rendimiento académico

\begin{tabular}{lll}
\hline & & $\begin{array}{l}\text { Rendimiento académico en } \\
\text { Inglés }\end{array}$ \\
\hline \multirow{3}{*}{ Clima familiar } & Correlación de Pearson & $0.778^{* *}$ \\
& Sig. (bilateral) & 0.000 \\
& $\mathrm{~N}$ & 90 \\
\hline
\end{tabular}

Según los resultados presentados en la tabla 2, se observa que si hay una relación positiva fuerte $(\mathrm{r}=0.778)$ y estadísticamente muy significativa $(* *)$ entre clima familiar y rendimiento académico en idioma inglés, esto indica que hay suficiente evidencia estadística para afirmar que cuanto mejor y muy favorable sea en clima familiar de los estudiantes, mayor será el rendimiento académico en idioma inglés, o de lo contrario cuanto menos favorable sea el clima familiar entonces el rendimiento académico será muy bajo.

\section{Tabla 3}

Coeficiente de correlación entre clima familiar y rendimiento académico

\begin{tabular}{|c|c|c|}
\hline & & $\begin{array}{l}\text { Rendimiento académico en } \\
\text { Inglés }\end{array}$ \\
\hline \multirow{3}{*}{$\begin{array}{l}\text { Dimensión } \\
\text { Relaciones } \\
\text { Familiares }\end{array}$} & Correlación de Pearson Sig. & $0.765^{* *}$ \\
\hline & (bilateral) & 0.000 \\
\hline & $\mathrm{N}$ & 90 \\
\hline \multirow{3}{*}{$\begin{array}{l}\text { Dimensión } \\
\text { Desarrollo } \\
\text { Familiar }\end{array}$} & Correlación de Pearson & $0.548^{* *}$ \\
\hline & Sig. (bilateral) & 0.000 \\
\hline & $\mathrm{N}$ & 90 \\
\hline Dimensión & Correlación de Pearson & $0.882^{* *}$ \\
\hline Estabilidad & Sig. (bilateral) & 0.000 \\
\hline Familiar & $\mathrm{N}$ & 90 \\
\hline
\end{tabular}


La tabla 3 muestra los coeficientes de correlación para las dimensiones de la variable clima familiar frente al rendimiento académico en el idioma inglés, aquí se pueden observar que si hay una relación positiva fuerte $(\mathrm{r}=0.765)$ y estadísticamente muy significativa $\left(^{* *}\right)$ entre la dimensión relaciones familiares del clima social y rendimiento académico en idioma inglés, asimismo hay una relación positiva media $(r=0.548)$ pero si es estadísticamente significativa (**) entre la dimensión desarrollo familiar del clima social y rendimiento académico en idioma inglés, y por último también hay una relación positiva fuerte $(r=0.882)$ y estadísticamente muy significativa $(* *)$ entre la dimensión estabilidad familiar del clima social y rendimiento académico en idioma inglés, estos resultados corroboran la contrastación de la hipótesis general.

\section{DISCUSIÓN}

De las tablas y figuras presentadas en la sección de los resultados se deduce que efectivamente existe una relación directa y significativa entre el clima familiar y rendimiento académico en idioma inglés, dichos resultados son corroborados con la investigación realizada por Chávez (2011) al concluir que existe una relación directa y significativa entre el clima familiar y el rendimiento académico de los estudiantes de la institución educativa "San Martín de Porres" de Yauli Huancavelica en el año 2011, al mismo se suman los aportes encontrados por Moreno (2009) cuando claramente indica que el clima familiar si se relaciona de manera directa con el clima social del aula, además de ello lo que viene de familia solo se reproduce en la escuela, aportes como los que permiten recomendar que se debe prestar mucha atención y mejorar el clima familiar, dado que ello es un indicador condicionante para lograr un buen rendimiento académico en el desarrollo de los estudiantes universitarios.

Por otra parte, también cabe mencionar a autores como Rosales (2013), Estevez (2008) y Gonzales y Pereda (2009) ya que todos ellos convergen en sus conclusiones indicando que el clima social familiar se encuentra asociada o relacionada al rendimiento escolar en los estudiantes de distintos niveles educativos, frente a ello también en la presente investigación se alcanzaron resultados similares, muy a pesar de existir variedad de climas dentro de las familias, así mismo en el rendimiento académico intervienen mucho factores como; la actitud del profesorado, comportamiento de los estudiantes, desarrollo de la empatía por parte de los estudiantes en el aula, inclusive las autoridades de la institución educativa.

\section{CONCLUSIONES}

Hay una relación positiva fuerte y, estadísticamente, muy significativa entre el clima familiar y el rendimiento académico en idioma inglés en estudiantes del Centro de Idiomas de la Universidad Nacional José María Arguedas de Andahuaylas durante el primer semestre 2018; ello permite afirmar que cuanto más favorable sea en clima familiar de los estudiantes en su familia, mayor será el rendimiento académico en idioma inglés, o de lo contrario cuanto menos favorable sea el clima familiar entonces el rendimiento académico será muy bajo.

Existe relación significativa entre las dimensiones relaciones familiares, desarrollo familiar y estabilidad familiar frente al rendimiento académico en los estudiantes del 
Centro de Idiomas de la Universidad Nacional José María Arguedas de Andahuaylas durante el primer semestre 2018, observándose una relación fuerte entre las dimensiones relaciones familiares y estabilidad familiar con el rendimiento académico, y hay una relación media entre la dimensión desarrollo familiar con el rendimiento académico en los estudiantes.

\section{REFERENCIA BIBLIOGRÁFICA}

Begoña, K. (2010) Percepción del clima familiar en toxicómanos. Tiene una influencia importante en todas las esferas de su desarrollo personaly social. Universidad de Cadiz, España.

Benites, L. (1997) Tipos de familia, habilidades, sociales y autoestima en un grupo de Adolescentes en situación de Riesgo. Lima. UPSMP. Revista Cultura No 12.

Chávez, C. (2011) tesis: Relación entre clima familiar y rendimiento académico de los estudiantes de la institución educativa "San Martín de Porres" de Yauli, Huancavelica - 2011. Escuela de Post Grado de la Universidad Nacional de Huancavelica.

Enciclopedia ENCARTA (2009) Programa multimedia. Microsoft.

Estevez, F (2008), Clima familiar, clima escolar y satisfacción con la vida en adolescentes, en la Universidad de Málaga España.

García, C. R. (2005). Habilidades Sociales, Clima Social Familiar y Rendimiento Académico en Estudiantes Universitarios. Liberabit. Revista de Psicología, número 011, Lima, Perú: Universidad de San Martín de Porres.

Gonzales y Pereda (2009) Relación entre el clima social familiar y el rendimiento escolar de los alumnos de la Institución Educativa No 86502 "San Santiago" de Pamparomás en el año 2006”. Trujillo, Perú: Escuela Internacional de Post Grado de la Facultad de Educación.

Lumman, P. (2006) Evaluación. Madrid: Ed. Paraninfo.

Ministerio de Educación (2009) Diseño Curricular Nacional. Lima: MINEDU.

Moos, R. H.; Moos, B.S. \& Tricket, E.J. (1989) Escala de Clima Social, Familiary de trabajo en Centros Escolares. Adaptación Española, Manual 3ra Edición, TEA Investigación y Publicaciones Psicológicas: Madrid.

Moreno, C. (2009) Relación entre el clima familiar y el clima escolar: el rol de la empatía, la actitud hacia la autoridady la conducta violenta en la adolescencia. Universidad de Valencia, España.

Novaez, M. (1986) Psicología de la actividad escolar. México: Ed. Iberoamericana.

Palomino, A. (2002) Familia y autoestima. Barcelona: Ariel.

Peñaloza, W. (2004) La educación integral. México: Ed. Iberoamericana.

Rosales (2013) La Percepción del Clima Familiar en Adolescentes Miembros de Diferentes Tipos de Familias. Universidad Autónoma de México.

Sloninsky, T. (1962). Familia y Relaciones Humanas. Buenos Aires. Ed. Ameba.

Solier, E. (2008) Evaluación contemporánea. Madrid: Ed. Paraninfo. 
Universidad Nacional Autónoma de Huanta

Suárez, T. (1998) Familia. Buenos Aires, Argentina: Ed. Ameba.

\section{CITAR COMO:}

Aroquipa Durán, Y., \& Sucari León, R. (2019). Clima familiar y rendimiento académico en estudiantes del Centro de Idiomas de la Universidad Nacional José María Arguedas - Andahuaylas 2018. Puriq, 1(01), 57-67. https://doi.org/10.37073/puriq.1.01.12 